\title{
GENETICAL CONTROL OF THE INCUBATION PERIOD IN MICE OF THE NEUROLOGICAL DISEASE, SCRAPIE
}

\author{
A. G. DICKINSON \\ A.R.C. Animal Breeding Research Organisation, Edinburgh \\ and \\ J. M. K. MACKAY \\ Moredun Institute, Gilmerton, Edinburgh
}

Received 29.xi.63

\section{INTRODUCTION}

ScRAPIE is a naturally occurring disease of adult sheep which is characterised clinically by signs of pruritis and locomotor inco-ordination. These signs are very variable especially from breed to breed and one or other may predominate. The disease is transmissible by inoculation of a wide variety of tissue extracts from affected animals. Pathologically the lesions appear to be confined to the central nervous system, where the process is essentially a degenerative one. Histological examination is necessary to demonstrate the changes, one of which is a characteristic intraneuronal vacuolation (Zlotnik, I962).

Study of the experimental disease in sheep has been hampered by the long incubation period before clinical signs occur-a minimum period of four months with no recognisable maximum duration-and the fact that only a variable proportion of sheep appear to be susceptible. There is also the possibility of the natural occurrence of the disease in sheep available for study. In contrast the great majority of goats appear to be susceptible to goat-adapted sources of scrapie. However, the economic limitations imposed on the use of large animals for bioassay purposes has made it difficult to adopt a quantitative approach to such problems as any biological variation in the agent or its resistance to physical or chemical treatment; for example, the disease can apparently be induced with boiled or formalin treated material. With sheep and goats, at present, there is little possibility of estimating, eliminating, or controlling any genetical variation in susceptibility.

Numerous attempts have been made during the past fifteen years to adapt the scrapie agent to mice: the first to succeed was Chandler (196I) who produced an encephalopathy in mice by inoculating with brain material from affected goats. Scrapie-like diseases have now also been transmitted to mice from a variety of scrapie sources, including directly from affected sheep (Zlotnik and Rennie, I962, 1963; Morris and Gajdusek, 1963; Dickinson and Mackay, unpublished).

The experiments described in this paper set out to assess the extent 
to which genetical variation might affect the response of mice to one strain of scrapie agent. Adaptation of scrapie to mice has presented the opportunity for critical bioassay which was not feasible with large animals. The optimum type of animal for this purpose is one with minimum response time, minimum genetic variability and maximum phenotypic stability to extraneous environmental variables. To this end the extent of genetical determination and the influence of the degree of inbreeding are considered in terms of a quantitative trait appropriate for bioassay purposes.

\section{EXPERIMENTAL MATERIALS AND METHODS}

(i) Details of scrapie agent: origin, symptoms and neuropothology

The agent, supplied by Dr I. Zlotnik, was from the M.E. 7 strain (Zlotnik and Rennie, 1963). This strain originated from brain and spleen of a natural scrapie Suffolk sheep being fed to mice of a random bred closed colony at Moredun Institute. The experiment is based on a pool of brains of affected Moredun-stock mice from the second passage by inoculation in these random bred mice. The incidence of scrapie has been ioo per cent. at all passages.

The reasons for preferring this particular strain of agent are twofold. Neuronal vacuolation is a prominent feature of the disease produced by this strain and the clinical syndrome is very clear-cut and in this respect more satisfactory than for other strains we have handled. A mouse showing the clinical syndrome, which commences three months or more after injection, passes through a short excitable phase with jerky movements, which is easily overlooked and difficult to score, into a prolonged phase of daytime inactivity in which the head is characteristically held rigid and extended: the animal is reluctant to move its head even if restraint is threatened by holding its head lightly. The entire body is in a permanent state of muscular tension and the righting-reflex is slowed down for affected mice placed on their back in the palm. Surprisingly, the affected mice during the daytimeinactive phase can be more active than controls during the night, as measured by a cage activity counter in operation from I I p.m. to $4 \mathrm{a} . \mathrm{m}$. It is impossible to generalise about loss of condition due to the disease-some strains endure four weeks or more in the inactive phase without losing condition, while others lose condition quickly and, consequently, are liable to die after two or three weeks. In the terminal phase of the disease there is typically a distended bladder and males have persistent priapism. Evidence of pruritis is entirely lacking and there is nothing in the excitable phase to suggest that pruritis is the cause of the excitability.

\section{(ii) Experimentol design}

Mice were weaned at weekly intervals when from three to four weeks old and inoculated intracranially two days after weaning: there were eight weaning groups. Weaned mice were stored six to a cage, the 
sexes being in different cages, and in any one cage there was usually only one and never more than two of each strain or cross. In the last four weaning groups uninjected controls were present and either one or two of the six mice could be a control but they bore no mark by which they could be distinguished immediately. Cage positions in the room were re-randomised at least once a month to counteract any effect of draughts, local heating, etc. The mouse room temperature was usually maintained in the range $20-22^{\circ} \mathrm{G}$., but there were occasional wide deviations from this due to mechanical failure.

\section{(iii) Mouse stocks and mating plan}

Five highly inbred stocks of mice and four partially inbred strains with a common random-bred origin have been used. The five standard inbred strains were chosen for their diversity and have been sib-mated for a further one to three generations since being received.

The inbred strains were SM/JaxBt, BSVS/Sr, RIII/FaBt, $\mathrm{C}_{57} \mathrm{BL} /$ $\mathrm{FaBt}$ and $\mathrm{LG} / \mathrm{JaxBt}$. In addition four inbred lines from the Moredun Institute closed colony have been sib-mated for three generations: these are designated $2 \mathbf{M}, 3 \mathbf{M}, 4 \mathbf{M}$ and $5 \mathbf{M}$. The experiment was designed to use the four $\mathbf{M}$ lines as a single semi-inbred group in crosses with other strains but the analysis has shown the need to treat them separately. Therefore the numbers of animals in mating blocks having $\mathbf{M}$ lines as one or both parents is unfortunately variable and several of the possible combinations are missing.

No tumours were seen in any of the experimental mice and there was no evidence of any infectious disease occurring which might have confused the experimental results. At the start of the experiment all animals were free from ectoparasites, but a few may have become reinfested with mites in the later stages.

The experiment was designed as a diallel cross-all possible crosses and purelines-and reciprocal crosses were included wherever the stock numbers permitted. The relative numbers of mice of the various types were largely controlled by the fertility and survival to weaning. Infertility of LG mice resulted in the absence of $\mathrm{SM} \times \mathrm{LG}$ crosses and this was the only missing block, apart from the complication with the $\mathrm{M}$ lines.

\section{(iv) Preparation of inocula}

The brains used as agent source were stored at $-20^{\circ} \mathrm{C}$. They were allowed to thaw twice: initially for division into sub-pools and finally when used. The brain suspensions for challenging the eight weaning groups were made up freshly for each group as a Io per cent. saline suspension using a tissue grinding tube. The suspension was allowed to sediment for varying periods and the clearer supernatant used as the inoculum. For the first weaning group the time allowed for the sediment to settle was less than $\frac{1}{2}$ hour and a high immediate death rate in this group was attributed to the presence of larger tissue fragments in the inoculum. For groups two to eight a two to three hour 
sedimentation period was used and there were no immediate deaths. With an intracranial route of injection in weanlings it is difficult to administer a predetermined dose: the approximate dose given was $0.02 \mathrm{ml}$. Only two experimental animals (neither likely to be genetic variants) have failed to develop scrapie out of 718 injected: it may be that these effectively received no inoculum. One contact control out of 72 developed scrapie at 49 weeks old and we tentatively consider that this event may be evidence of contagious spread. At the time of writing the average age of control animals is 43 weeks. However, it remains to be seen whether contact transmission can occur since prolonged contact with clinical cases in sheep was necessary for transmission to occur to goats (Stamp, 1962).

\section{(v) Clinical criteria}

All weaned mice were scored for clinical symptoms of scrapie weekly, during the afternoon, and were weighed at the same time. The scoring system depended entirely on observation of the daytimeinactive phase and each mouse was scored as either " unaffected", "possibly affected" or "affected". Animals were killed after the third consecutive score of "affected". Records were designed to prevent knowledge by the operator of the animals' previous scores and mice were always handled in random order. As an indication of the repeatability of this scoring system there were only $\mathrm{I} \cdot 3$ per cent. of occasions when an "unaffected" score was given to an animal which had previously been scored as " affected" while the rate of remissions from " affected" to " possibly affected" was 7.5 per cent.

A number of animals died before the third consecutive " affected" score was reached. In these cases the actual age at death was used in the analysis instead of the intended observation of age at third "affected" score. The overall proportion of mice dying early was I I per cent. The standard inbreds probably succumb quicker than the $\mathrm{F}_{1}$ 's judging from the following figures for mice dying early: $F_{1}$ 's 9 per cent.; standard inbreds 24 per cent. (if the LG strain is excluded this figure becomes I 7 per cent.). It should not be concluded from these results that the clinical diagnosis was unreliable for animals which did not survive till the third "affected" score because very sick animals were kept under daily surveillance: these observations, apart from confirming the clinical syndrome, are not used in the analysis.

Forty brains of affected animals were chosen at random for pathological examination of the brain as a check on the clinical scorings: all were confirmed positive and we are indebted to Dr Zlotnik for this assistance.

\section{RESULTS}

The analysis is confined to data on the incubation periods, the term being used here to denote the interval from inoculation to killing as defined above. It is recognised that the incubation period here is 
confounded with age, since all animals were inoculated at the same age.

Reciprocal crosses were included for formal completeness of the design and not because they were expected to reveal any source of variation. In all, there are fifteen pairs of reciprocals, treating the sexes separately, of which none show significant differences. Consequently, reciprocals have been pooled.

Also there is no evidence for an overall difference between the sexes which is somewhat surprising but we have some limited observations which lead us to suspect that a sex difference may have emerged if age at death had been used rather than an arbitrary killing criterion: males appear to lose condition very rapidly in the terminal phases in comparison with the females as shown by observation of their weights.

Reasons have already been given for expecting differences between the eight sub-pools of inoculum at least in the case of the first inoculum. However, there is no statistically significant evidence for variation between any of the inocula as shown by the following analysis of variance. There are too many missing combinations to test inoculum differences within each strain or cross, therefore the genotype differences have been reduced to eight (omitting $2 \mathrm{M}$ and $3 \mathrm{M}$ ) by pooling all crosses with the parental strain they have in common and with the individual crosses weighted by the reciprocal of their variance. Even so about ro per cent. of the values are missing and these have been estimated by fitting constants to give a complete table of eight inocula $\times$ eight genotypes. Using the interaction term as an estimate of error, the variance ratio for inocula is not significant (V.R., I $89 ; P>0 \cdot I$ ) in contrast with highly significant differences between genotypes (V.R., 15.41; P<0.001).

\section{GENETICAL ANALYSIS}

A summary of the results is given in table $\mathrm{I}$ : sex, inoculation groups and reciprocal crosses are pooled for each strain or cross.

There is no satisfactory indication that the scale of measurement is inadequate for the genetical analysis. For the fully inbred strains and their $\mathrm{F}_{1}$ 's there is no correlation of mean and variance $(r=0.02)$ and the only evidence of such a correlation comes from a consideration of the eight purebred strains alone $(r=0.83)$. However, the two groups with the highest means $\left(2 \mathrm{M}\right.$ and $\left.{ }_{5} \mathbf{M}\right)$ are also the ones known to be least inbred and it is reasonable to attribute some of their variability to genetic heterogeneity: exclusion of $5 \mathrm{M}$ results in a nonsignificant low correlation for the remaining seven.

There is one missing block in the main $7 \times 7$ series of crosses, namely $\mathrm{SM} \times \mathrm{LG}$ : since the two parental strains have almost identical mean values an intermediate value of $21 \cdot 9$ weeks has been used in the analysis to simplify presentation. Unless there is an unpredictable genetic interaction in this particular cross this interpolation will lead to slight underestimation of the average degree of dominance of the SM strain. 
A. G. DICKINSON AND J. M. K. MACKAY

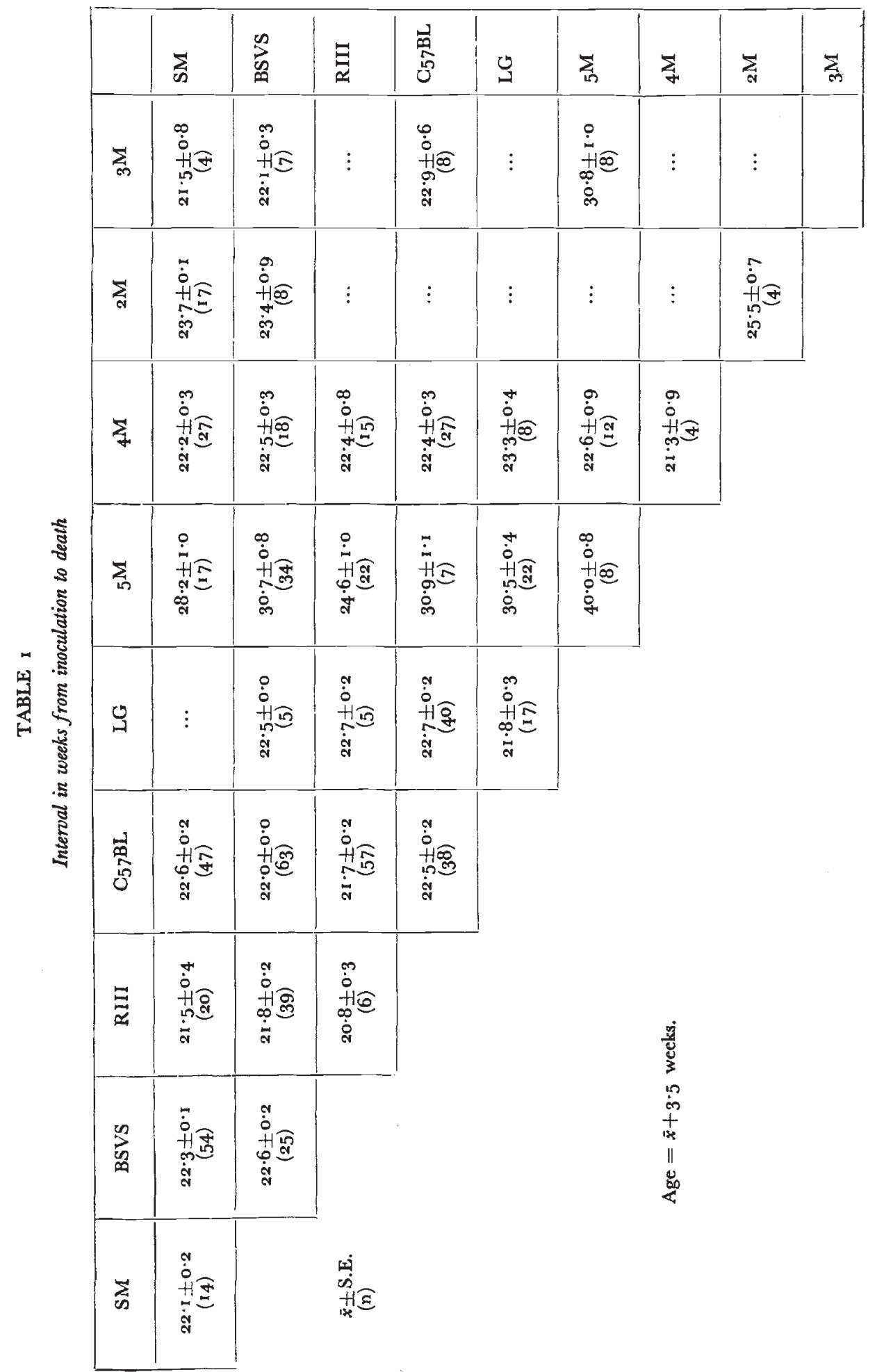


In view of the deliberate choice of standard inbred strains differing widely from one another it is surprising that they display such close similarities in response to scrapie. RIII seems to succumb slightly quicker than the four others. In contrast the $2 \mathrm{M}, 4 \mathrm{M}$ and ${ }_{5} \mathrm{M}$ strains despite small numbers per group, show significant and marked differences from each other. Almost two-thirds of the strains or crosses involving the four $\mathrm{M}$ lines have higher variances than the most variable of the ones involving the other five inbred lines. Since the four $\mathbf{M}$ lines were only $5^{\circ}$ per cent. inbred compared with the long history of sib mating in the five standard strains, it is presumed that the variability with the M's is evidence of residual heterozygosity.

It would be reasonable to suspect that the difference in incubation period of the $\mathrm{M}$ lines, particularly ${ }_{5} \mathrm{M}$, from the rest might diminish with further inbreeding as an outcome of inbreeding depression. This seems to be unlikely as there is no evidence of such depression having occurred in the five standard inbreds, judging from the performance of their $F_{1}$ 's. In addition, there is no evidence that these $F_{1}$ 's have lower intragroup variances than their parents.

The genetical analysis employed is that derived by Dickinson and Jinks (1956) and applicable to the interpretation of diallel crosses where some parents are not fully inbred. The comparison is used of the variance, $V_{r}$, of an array (all the mating types which have one parental strain in common) with the covariance $\mathrm{W}_{\mathrm{r}}$, between that array and the parental strains. The relationship of $V_{r}$ and $W_{r}$ is shown in fig. 1: all points must lie inside the parabola shown. The effect of a strain being partially heterozygous is that its average degree of dominance is underestimated.

Some of the criteria used in interpretation are indicated on fig. I. On average, $\mathrm{C}_{57}$ and BSVS display no dominance and the same is almost true for LG. SM shows a small degree of dominance having practically all the slightly dominant genes; much the same applies to RIII but at a much higher degree of partial dominance. Likewise, in view of its position almost on the parabola and near the origin, ${ }_{4} \mathrm{M}$ appears to have virtually all the dominant genes but in this case there is at least full dominance. Finally, for ${ }_{5} \mathrm{M}$ the genetic constitution is very different from the rest; there is a preponderance of recessive genes and the gene action in this strain shows an almost full degree of dominance, which is likely to be underestimated because inbreeding is incomplete.

As judged by the intragroup variances of the inbred lines, the variances of the $4 \mathrm{M}$ strain and its crosses are relatively low for a strain which is only partially inbred and this is as expected from the above interpretation of a very high proportion of fully dominant genes in ${ }_{4} \mathrm{M}$. In contrast the intragroup variances of the $5 \mathrm{M}$ strain and its crosses are all high which again accords with the interpretation given above: namely, that there is considerable residual heterozygosity, in which fully dominant alleles are in the minority. 


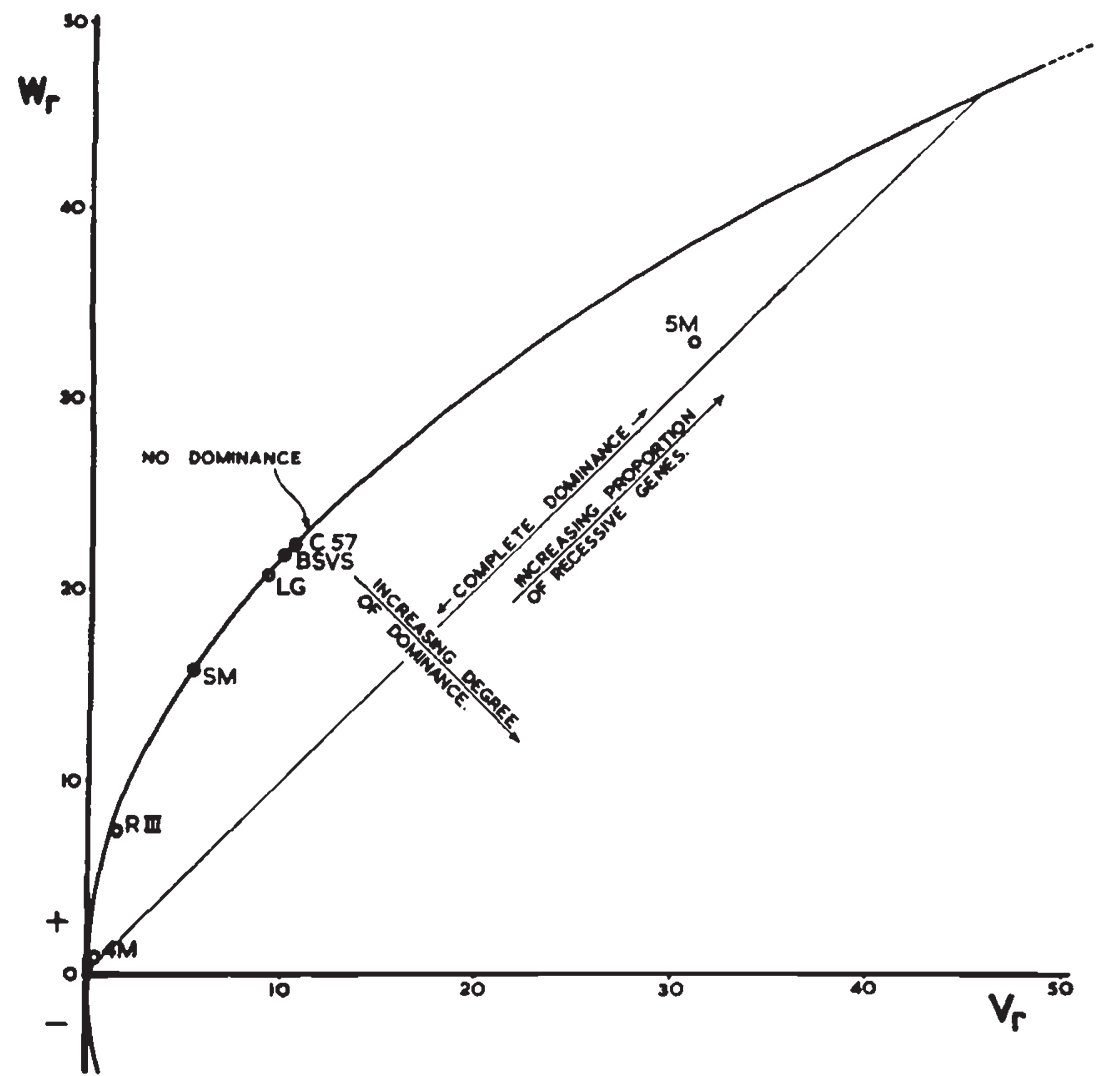

Fig. 1.-Relationship between array variance, $\mathrm{Vr}$, and covariance of array with nonrecurrent parents, $\mathrm{Wr}$, for each mouse strain.

TABLE 2

Variance and Covariance of arrays for " replicates"

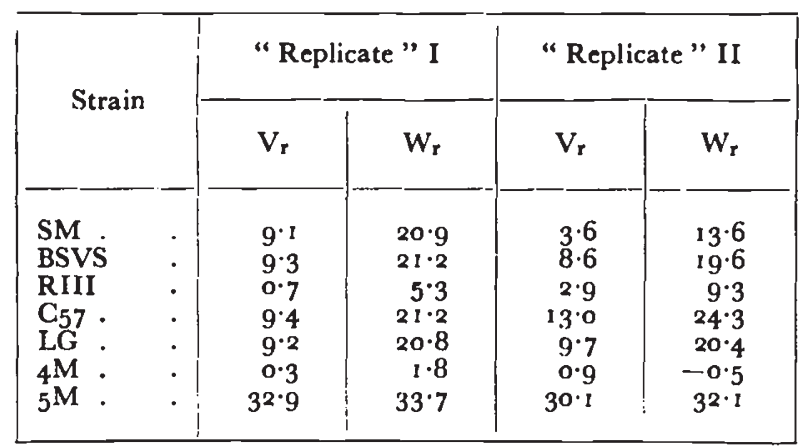

The reliability of the information on which fig. I is based could have been estimated if the experiment had been replicated. As this was not done, an indication of reliability can be deduced from artificial replication of the experiment by subdividing each mating type and analysing the two half experiments separately. Many methods of subdivision could have been used, the one chosen was to reallocate 
animals by order of birthdate alternately into the two sub-groups: the replicate $V_{r}$ and $W_{r}$ values which result are given in table 2 . Only in the case of the SM strain is there any reason for doubting the interpretation given above.

\section{DISCUSSION}

The particular strain of scrapie we have used, appears to have high potential for bioassay purposes in any one of these standard inbred strains and there seems to be no intrinsic value in resorting to the use of $F_{1}$ crosses. The type of bioassay applications envisaged relate to understanding the effect of physical and chemical treatment of this remarkably resistant disease agent. By scoring the clinical disease twice weekly and using groups of 20 mice, it should be easy to distinguish 5 per cent. differences in incubation period. It remains to be shown that change in concentration or structure of the agent used here can be detected as a change in incubation period but there is some evidence from other work to encourage this expectation (Pattison and Smith, I963).

Although this work has been carried out using clinical criteria alone, we have no illusions about the difficulties of using solely clinical criteria in most scrapie studies and we fully expect that the considerable effort involved in adopting pathological scoring would have added much to the delicacy of our tests--the duration of the test could probably have been reduced by a quarter.

It appears likely that there is scope for further selection within the $5 \mathrm{M}$ line and a rough estimate based on the diallel analysis and the phenotypic variance is that the incubation period could be increased to at least 45 weeks, without any further mutation, simply by accumulating a full complement of recessives and leaving untapped any heterozygosity for genes which show no dominance. The variation between lines could therefore be increased even more than the present twofold difference. The magnitude of the differences between lines can be brought into perspective by considering them as proportions of an average mouse's lifespan: the most rapid incubation period is about a quarter of a lifetime, the slowest about half of a lifetime. Though difficult to attain in practice, it is easy to visualise selection for increased incubation period reaching a point where the animals dic naturally before developing scrapie.

It is interesting to consider some implications of these findings for current research on scrapic, particularly in sheep. At present, we find it necessary to keep experimentally challenged sheep for about two years after inoculation to discriminate susceptible individuals from resistant ones. In view of the evidence presented here, this policy may need to be revicwed to avoid classifying sheep as " unaffected "if a longer interval had been allowed for. The same applies to work on natural scrapie in shecp but the tactical implications are even more sobering as the incubation period is much longer. Put in 
general terms, the evidence here calls in question any easy application of the concept of absolute "resistance", in its commonly accepted meaning of failure to develop a disease, where a chronic illness of long incubation period is concerned.

\section{SUMMARY}

I. Data are presented for a diallel cross involving a wide variety of mouse strains. All animals were challenged at weaning by intracranial injection with a mouse-adapted scrapie agent. The incidence of scrapie was virtually roo per cent. in all strains and crosses.

2. There was a very wide range of incubation periods between strains extending from 20 to 40 weeks and this aspect of the disease is evidently under a high degree of genetic control.

3. No difference in incubation period was detected between sexes or reciprocal crosses.

4. Non-additive genetic variation, interpreted as dominance, is shown to affect incubation period in some stocks.

5. There is evidence of scope for increasing the range of incubation period between strains by selection within some of the partially inbred stocks.

6. The phenotypic variation of incubation period was alike for inbreds and first-crosses, so that for bioassay purposes there is no intrinsic disadvantage in using these inbred stocks in preference to first-crosses. Bioassay differences of one week in incubation period should be readily detectable using the techniques described.

7. The concept of absolute resistance to disease is questioned in the light of the findings.

Acknowledgments.-It is a pleasure to record our thanks to Miss Veronica HamiltonMeikle for her skilful administration of the practical aspects of the experiment.

This work was carried out with a grant from the United States Department of Agriculture administered under PL 480 .

\section{REFERENCES}

CHANDLER, R. L. 1961. Encephalopathy in mice produced with scrapie brain material. Lancet, $i$, 1378-1379.

Dickinson, A. G., AND JiNKs, J. L. 1956. A generalised analysis of diallel crosses. Genetics, $4 I, 65-78$.

MORRIS, J. A., AND GAJDUSEK, D. c. I963. Encephalopathy in mice following inoculation of scrapie sheep brain. Nature, 197, 1084-1086.

PATTison, I. H., AND SMiTh, K. I963. Histological observations on experimental scrapie in the mouse. Res. Vet. Sci., 4, 269-275.

Stamp, J. T. I962. Scrapie: a transmissible disease of sheep. Vet. Rec., 74, 357-362. zLOTNIK, I. I962. The pathology of scrapie: a comparative study of lesions in the brain of sheep and goats. Acta Neuropath. Suppl. I, 6r-70.

ZLOTNIK, I., AND RENNIE, J. C. 1962. The pathology of the brain of mice inoculated with tissues from scrapie sheep. 7. Comp. Path., 72, 360-365.

zLOTNIK, I., AND RENNIE, J. c. 1963. Further observations on the experimental transmission of scrapie from sheep and goats to laboratory mice. F. Comp. Path., $73,150-162$. 